ARTICLE

\title{
Persistent and reversible solid iodine electrodeposition in nanoporous carbons
}

\author{
Christian Prehal (1) 1,8凶 , Harald Fitzek ${ }^{2}$, Gerald Kothleitner (10) 2,3, Volker Presser (1) 4,5, Bernhard Gollas (1) ${ }^{1}$, \\ Stefan A. Freunberger (10 ${ }^{1,6 凶} \&$ Qamar Abbas (1) ${ }^{1,7 凶}$
}

Aqueous iodine based electrochemical energy storage is considered a potential candidate to improve sustainability and performance of current battery and supercapacitor technology. It harnesses the redox activity of iodide, iodine, and polyiodide species in the confined geometry of nanoporous carbon electrodes. However, current descriptions of the electrochemical reaction mechanism to interconvert these species are elusive. Here we show that electrochemical oxidation of iodide in nanoporous carbons forms persistent solid iodine deposits. Confinement slows down dissolution into triiodide and pentaiodide, responsible for otherwise significant self-discharge via shuttling. The main tools for these insights are in situ Raman spectroscopy and in situ small and wide-angle X-ray scattering (in situ SAXS/WAXS). In situ Raman confirms the reversible formation of triiodide and pentaiodide. In situ SAXS/WAXS indicates remarkable amounts of solid iodine deposited in the carbon nanopores. Combined with stochastic modeling, in situ SAXS allows quantifying the solid iodine volume fraction and visualizing the iodine structure on 3D lattice models at the sub-nanometer scale. Based on the derived mechanism, we demonstrate strategies for improved iodine pore filling capacity and prevention of self-discharge, applicable to hybrid supercapacitors and batteries.

\footnotetext{
${ }^{1}$ Institute for Chemistry and Technology of Materials, Graz University of Technology, Stremayrgasse 9, 8010 Graz, Austria. ${ }^{2}$ Graz Centre for Electron Microscopy, Steyrergasse 17, 8010 Graz, Austria. ${ }^{3}$ Institute of Electron Microscopy and Nanoanalysis, NAWI Graz, Graz University of Technology, Steyrergasse 17, 8010 Graz, Austria. ${ }^{4}$ INM - Leibniz Institute for New Materials, Campus D2 2, 66123 Saarbrücken, Germany. ${ }^{5}$ Department of Materials Science and Engineering, Saarland University, Campus D2 2, 66123 Saarbrücken, Germany. ${ }^{6}$ IST Austria (Institute of Science and Technology Austria), Am Campus 1, 3400 Klosterneuburg, Austria. ${ }^{7}$ Institute of Chemistry and Technical Electrochemistry, Poznan University of Technology, Berdychowo 4, 60-965 Poznan, Poland. ${ }^{8}$ Present address: Department of Information Technology and Electrical Engineering, ETH Zürich, Gloriastrasse 35, 8092 Zürich, Switzerland.

凶email: cprehal@ethz.ch; stefan.freunberger@ist.ac.at; qamar.abbas@tugraz.at
} 
$\mathrm{R}$ educing the ecological and economic footprint of electrochemical energy storage requires battery and storage concepts beyond standard intercalation-type Li-ion batteries. Current technologies suffer from the need of expensive, toxic, and flammable materials that are often obtained under harsh environmental and socioeconomic conditions ${ }^{1}$. Amongst the possible alternatives, iodine-based aqueous systems, such as iodide hybrid supercapacitors ${ }^{2-5}$, zinc iodine batteries ${ }^{6-8}$, or zinc iodide flow batteries ${ }^{9,10}$ are highly promising considering their performance, sustainability, and environmental aspects. However, to see their more widespread use in mobile or stationary applications, energy density, rate capability, and longterm stability need to become competitive with current Li-ion battery technology.

The critical process determining the performance of both aqueous $^{2,8,11}$ and organic ${ }^{12-15}$ iodine-based energy storage is the reversible oxidation/reduction of iodide/iodine at $0.54 \mathrm{~V}$ vs. standard hydrogen electrode (SHE). At a planar platinum surface, triiodide $\left(\mathrm{I}_{3}{ }^{-}\right)$forms by electrochemically oxidizing iodide $\left(\mathrm{I}^{-}\right)$to iodine $\left(\mathrm{I}_{2}\right)$, followed by comproportionation of $\mathrm{I}^{-}$and $\mathrm{I}_{2}{ }^{16,17}$. In aqueous hybrid supercapacitors and iodine batteries, the reaction occurs in the nanopores of positively polarized carbon electro$\mathrm{des}^{2-4}$. Confinement in such electrodes may change rates of individual reaction steps and hence the stability of $\mathrm{I}_{2}$ and $\mathrm{I}_{3}{ }^{-}$. While the polyiodides $\mathrm{I}_{3}{ }^{-}$and $\mathrm{I}_{5}{ }^{-}$are generally accepted to form during $\mathrm{I}^{-}$oxidation, described mechanisms are ambiguous $2,3,7,8,18-20$. Works on carbon-iodine battery cathodes ${ }^{15,21}$ suggest that the physico-chemical mechanism during iodide/ iodine oxidation/reduction is similar in both organic and aqueous electrolytes. The rather slow self-discharge of iodine-based electrochemical energy storage devices is currently attributed to immobile $\mathrm{I}_{3}{ }^{-}$and $\mathrm{I}_{5}{ }^{-}$confined in the narrow carbon pores of below $1 \mathrm{~nm}$ diameter ${ }^{7,8,18}$. However, given the known reaction mechanism on planar platinum electrodes ${ }^{16,17}$ and the use of battery electrodes with physically impregnated $\mathrm{I}_{2} 6,7$, alternative electrodeposition of solid $\mathrm{I}_{2}$ in carbon nanopores ought to be considered.

Resolving the mechanism requires in situ techniques with chemical and structural sensitivity for all involved species in the nanoporous carbon. In situ Raman spectroscopy probes species in electrochemical cells ${ }^{22-24}$ and is sensitive to polyiodides $\left(\mathrm{I}_{3}^{-}, \mathrm{I}_{5}^{-}\right)^{25-28}$. In situ small and wide-angle X-ray scattering (SAXS/WAXS) is sensitive to concentration changes and structural arrangements of molecules and ions in the nanoporous system ${ }^{29-32}$. Given the high scattering power of all iodine species $\left(\mathrm{I}^{-}, \mathrm{I}_{3}^{-}, \mathrm{I}_{5}^{-}, \mathrm{I}_{2}\right)$, in situ SAXS/WAXS data should provide rich kinetic and structural information. However, the complexity induced by the multiphase character of these systems makes the SAXS data analysis highly challenging ${ }^{29}$.

Here, we show that $\mathrm{I}^{-}$oxidation in microporous carbon (pore size $<2 \mathrm{~nm}$ ) produces solid $\mathrm{I}_{2}$, which can reach pore fillings of at least $30 \%$ and which in the iodide electrolyte partly dissolves into $\mathrm{I}_{3}{ }^{-}$and $\mathrm{I}_{5}{ }^{-}$. The latter are responsible for self-discharge via shuttling. In situ Raman and ex situ ultraviolet-visible (UV-vis) spectroscopy data confirm $\mathrm{I}_{2}, \mathrm{I}_{3}{ }^{-}$, and $\mathrm{I}_{5}{ }^{-}$during positive polarization and their reduction into $\mathrm{I}^{-}$during negative polarization. In situ SAXS/WAXS data show that most of these species is confined to the carbon nanopores. Combined with stochastic modeling, in situ SAXS quantifies the amount of solid iodine deposit and visualizes its structural evolution in the pores. Based on the derived reaction mechanism, we show that high capacity with low self-discharge requires a small concentration of mobile polyiodides and a large fraction of immobile iodine deposits.

\section{Results}

In situ Raman and SAXS/WAXS were done in two-electrode hybrid supercapacitor cells comprising activated carbon (AC) electrodes and aqueous $1 \mathrm{M} \mathrm{NaI}$ electrolyte. The iodide/iodine redox reaction takes place at the positive electrode, while the negative electrode stores charge exclusively via electrical doublelayer capacitance. Faradaic capacity (current) at the positive electrode would be limited by the capacitance (current) of an equally sized negative electrode ${ }^{33}$. We increased the amount of iodine within the positive electrode by oversizing the negative AC electrode about 10-times in mass to exploit the Faradaic capacity and enhance the in situ Raman and SAXS/WAXS signals. The microporous $\mathrm{AC}$ has a mean pore size of $0.81 \mathrm{~nm}$, a specific surface area of $1763 \mathrm{~m}^{2} \mathrm{~g}^{-1}$, and a specific pore volume of $0.84 \mathrm{~cm}^{3} \mathrm{~g}^{-1}$ (Supplementary Table 1 and Supplementary Fig. 1). The experimental set-ups and cell assemblies are discussed in the Methods and sketched in Supplementary Fig. 2.

In situ Raman spectroscopy. In situ Raman data were measured during cell voltage sweep between $-0.25 \mathrm{~V}$ and $+0.55 \mathrm{~V}$ at a scan rate of $0.32 \mathrm{mV} \mathrm{s}^{-1}$ (Fig. 1a). At negative voltages, the cell shows pure double-layer capacitance, with a current limited by the capacitance of the positive electrode. Upon positive polarization, $\mathrm{I}^{-}$is oxidized at the positive electrode with a significantly enhanced current, which is limited by the capacitive current of the oversized negative electrode. The negative current of virtually the same magnitude during negative polarization above $0 \mathrm{~V}$ indicates a high coulombic efficiency of the reversible Faradaic iodide/iodine reaction.

The Raman intensity as a function of time for two voltage cycles shows fully reversible formation/disappearance of $\mathrm{I}_{3}{ }^{-}$and $\mathrm{I}_{5}^{-}$at Raman shifts of $110 \mathrm{~cm}^{-1}$ and $165 \mathrm{~cm}^{-1}$, respectively (Fig. $1 \mathrm{~b}, \mathrm{c})^{26,27}$. The band forming around $224 \mathrm{~cm}^{-1}$ is an overtone of the $\mathrm{I}_{3}{ }^{-}$band $^{28}$. At larger Raman shifts, changes in the carbon-related G- and D-band intensity (band width and position) can be observed (Fig. 1d, Supplementary Fig. 3). With increasing cell voltage, the widths of the G- and D-modes increase, while they are slightly red- and blue-shifted, respectively and the $I_{\mathrm{D}}$-to- $I_{\mathrm{G}}$ ratio is lowered. This can be explained by a superposition of charge transfer and an increase of defects ${ }^{34-37}$, which is qualitatively consistent with ex situ results reported earlier ${ }^{18,38}$.

As positive polarization proceeds, initially dominant $\mathrm{I}_{3}{ }^{-}$band intensity gives way to the dominance of $\mathrm{I}_{5}{ }^{-}$band intensity (Fig. 1e, $\mathrm{f}$, curves from red to blue). Note that the $\mathrm{I}_{3}{ }^{-}$and $\mathrm{I}_{5}{ }^{-}$ band intensities do not directly correlate with their concentration, since the scattering cross-sections of the polyiodides may vary significantly (resonant vs. non-resonant scattering) ${ }^{39}$. To quantify the Raman band intensity changes, we deconvolute the Raman spectra as shown in Supplementary Fig. $4^{27}$. The band intensities as a function of the cell voltage (Fig. 1f) show an increasing $\mathrm{I}_{5}{ }^{-}$to $\mathrm{I}_{3}{ }^{-}$band intensity ratio with increasing cell voltage. Hence, relative to $\mathrm{I}_{3}{ }^{-}, \mathrm{I}_{5}{ }^{-}$forms at accelerated rates at higher cell voltages.

To check whether $\mathrm{I}_{2}$ had formed (next to $\mathrm{I}_{3}{ }^{-}$and $\mathrm{I}_{5}{ }^{-}$), we soaked charged (positively polarized) and washed AC electrodes for several minutes in either pure water or $1 \mathrm{M} \mathrm{NaI}$ and recorded ex situ UV-vis spectra (Fig. 1g, details in Methods). While pure water would leave $\mathrm{I}_{2}$ confined to the AC nanopores, it should be dissolved in the $1 \mathrm{M} \mathrm{NaI}$ solution via $\mathrm{I}_{2}+\mathrm{I}^{-} \rightleftharpoons \mathrm{I}_{3}^{-}$. Indeed, the 1 $\mathrm{M}$ NaI solution turned brownish, while pure water remained colorless (Supplementary Fig. 5). The UV-vis spectra in Fig. 1g confirm a large quantity of $\mathrm{I}_{3}{ }^{-}$in the $1 \mathrm{M} \mathrm{NaI}$ solution and hence the presence of confined $\mathrm{I}_{2}$ in the charged positive AC electrode ${ }^{40}$. 
a

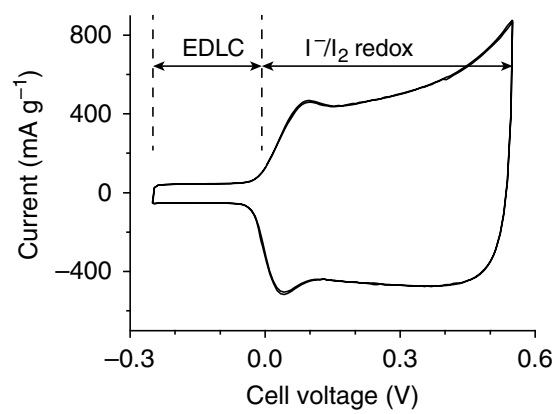

e

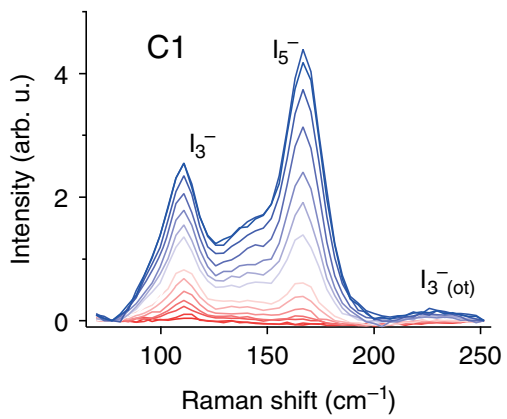

b Current $\left(\mathrm{mA} \mathrm{g}^{-1}\right)$

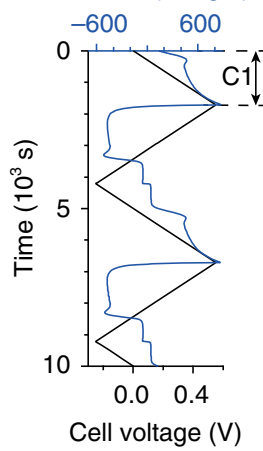

C

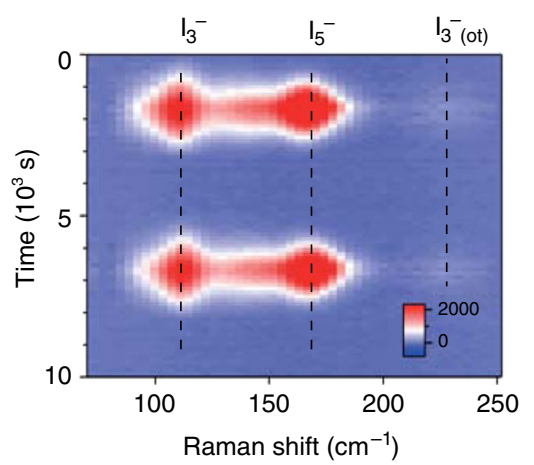

d

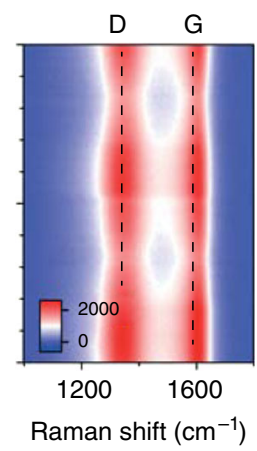

f

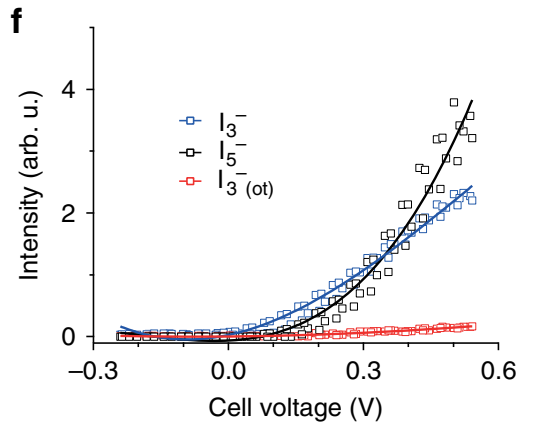

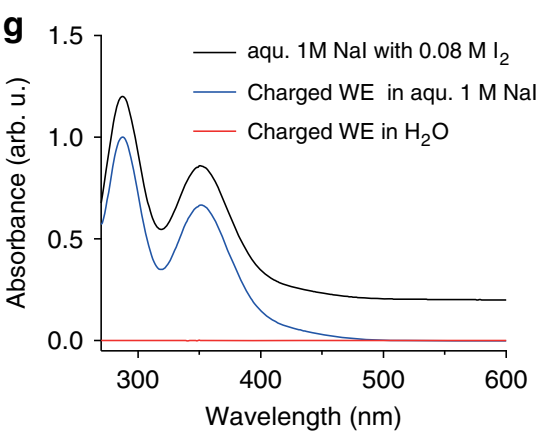

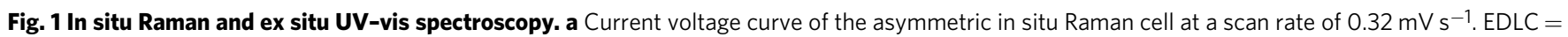
region of electrical double-layer capacitance as the predominant charge-storage process. $\mathbf{b}$ Cell voltage and current versus time for two full cycles. $\mathbf{c}$, $\mathbf{d}$ The corresponding Raman intensity as a function of time and Raman shift to track $\mathrm{I}_{3}-$ and $\mathrm{I}_{5}-$ bands (c), as well as the activated carbon (AC) G- and D-bands (d). e Raman spectra recorded during the first positive voltage sweep (C1). Colors change from red to blue with increased voltage. $\mathbf{f} \mathrm{I}_{3}{ }^{-}$and $\mathrm{I}_{5}{ }^{-}$band intensities as a function of cell voltage. Solid lines represent third order polynomial fits to serve as a guide to the eye. $\mathbf{g} \mathrm{UV}$-vis absorption spectra of a $1 \mathrm{M}$ Nal solution with $0.08 \mathrm{M} \mathrm{I}_{2}$ (black), $1 \mathrm{M} \mathrm{Nal}$ (blue), and deionized $\mathrm{H}_{2} \mathrm{O}$ where the washed, positively polarized (charged) carbon working electrode was soaked (red). Spectra are offset for clarity. More details and photographs of the measured solutions are given in Supplementary Fig. 5.

As an independent proof, we detected iodine after immersing the charged positive AC electrode for several minutes in ethanol, which directly dissolves $\mathrm{I}_{2}$ (Supplementary Fig. 5) ${ }^{41}$.

In situ small and wide angle $\mathrm{X}$-ray scattering. In situ Raman and ex situ UV-vis provide evidence for the formation of polyiodides $\left(\mathrm{I}_{3}{ }^{-}, \mathrm{I}_{5}{ }^{-}\right)$and $\mathrm{I}_{2}$ during $\mathrm{I}^{-}$oxidation. However, these methods fail to quantify the absolute amount of the species, to locate them in the nanopores, and to clarify whether $\mathrm{I}_{2}$ is a solute or solid. In situ SAXS/WAXS can afford all this (Fig. 2).

As before, we use an asymmetric two-electrode cell with oversized negative electrode and ran cell voltage sweeps with the scan rate controlled by the total cell voltage. The cell exhibits pure double-layer capacitance limited by the capacitance of the positive AC electrode at cell voltages below $+0.2 \mathrm{~V}$ (Fig. 2a). Above this voltage, $\mathrm{I}^{-}$is oxidized at the positive AC electrode, leading to a significantly enhanced current (in line with electrochemical data of the in situ Raman measurements). The X-ray transmission of the electrolyte-filled positive AC electrode was simultaneously recorded by a photodiode placed behind the in situ cell, and quantifies the material flux in and out of the positive AC electrode (Fig. 2c). The significant increase of the X-ray attenuation (details in ref. ${ }^{42}$ ) indicates the accumulation of large amounts of iodide, iodine, and polyiodides within the AC pores. The relative scattering intensity as a function of time follows the applied voltage and resulting current (Fig. 2b, d). During positive polarization, the scattering intensity in the SAXS regime at $q<5 \mathrm{~nm}^{-1}$ and in the WAXS regime has a clear maximum at the peak voltage.

Before analyzing the scattering intensity changes during positive cell polarization, we first must consider the data from the ex situ SAXS of the empty and electrolyte filled nanoporous carbon (Fig. 2e). The scattering intensity of the nanoporous carbon can be split into three additive terms: one accounting for spatial correlations on the molecular level, called structure factor, a second one accounting for the spatial correlations of the nanopores, which we refer to as nanopore scattering, and a third one accounting for the scattering contribution of the AC particles (with a size around $1 \mu \mathrm{m})^{31,43,44}$. Given the reciprocal relationship between the size of real-space objects and their appearance on the scattering curve, the structure factor contribution is most prominent at large scattering vectors $q$ (large scattering angles). The nanopore scattering appears as a distinct intensity hump at intermediate scattering vectors $q$, with the shape and the position containing information about the pore morphology, size distribution, and mean pore size. The increased scattering intensity at $q<0.8 \mathrm{~nm}^{-1}$ is caused by the scattering of the AC particles. The nanopore scattering intensity depends on the squared electron density difference between pores and carbon skeleton; therefore, filling of the nanopores with electrolyte leads to a significant decrease of the scattering intensity (Fig. 2e, inset). At the same time, the electrolyte structure factor increases the scattering intensity in the WAXS regime. The distinct intensity peak around $20 \mathrm{~nm}^{-1}$ can be attributed to the first water structure factor peak (Supplementary Fig. 6).

Qualitatively, the large SAXS/WAXS intensity increase at low and large scattering vectors $q$ (Fig. $2 \mathrm{f}$, red to blue for increasing polarization) can be assigned to different contributions. The distinct intensity hump around $3 \mathrm{~nm}^{-1}$ has a shape comparable to the nanopore scattering contribution of the empty AC shown in Fig. 2e. This can only be explained by large amounts of a high-density 
a

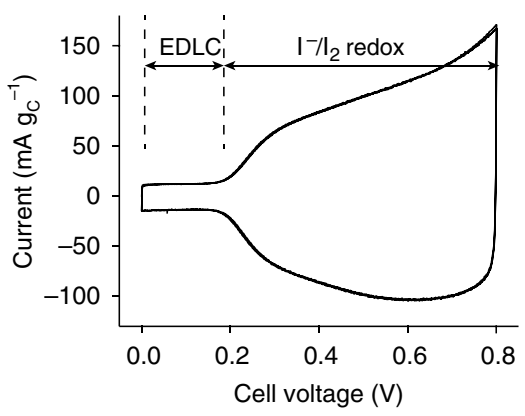

e

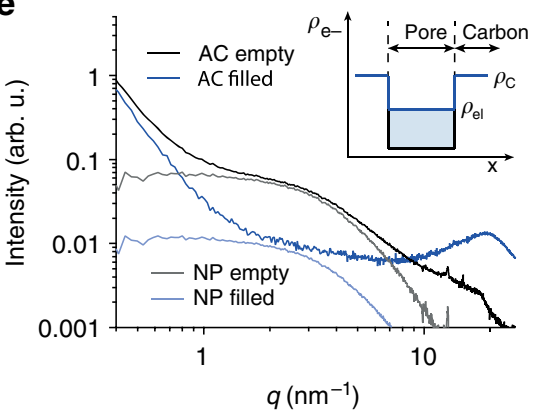

b

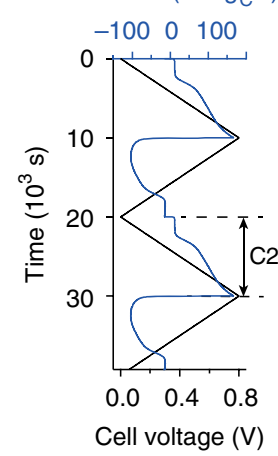

C

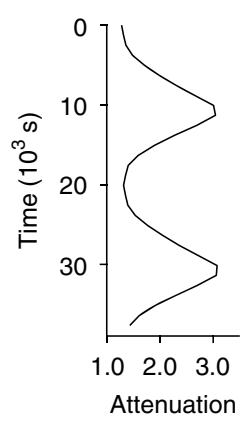

d

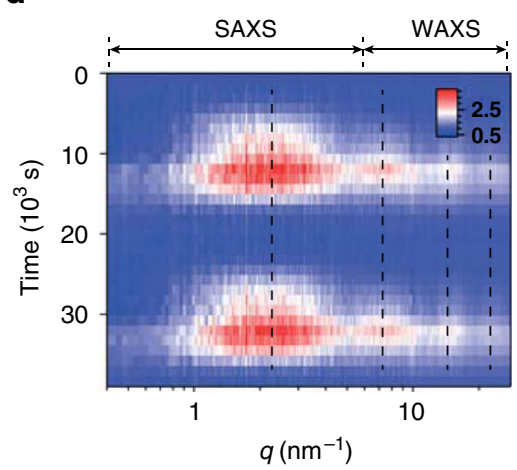

f

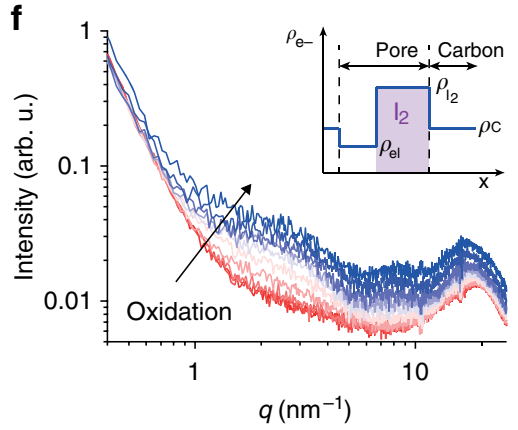

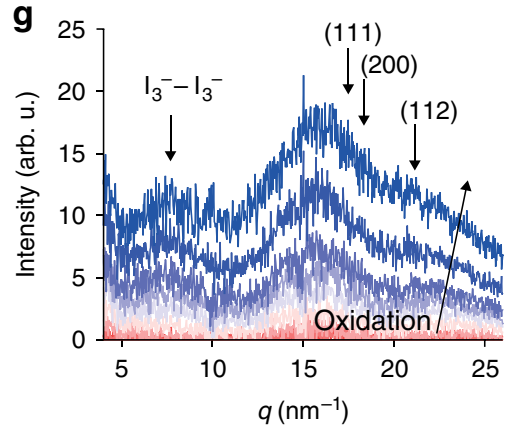

Fig. 2 In situ small and wide-angle X-ray scattering. a Current voltage curve of the asymmetric in situ SAXS/WAXS cell at a scan rate of $0.08 \mathrm{mV} \mathrm{s}^{-1}$. b Current and cell voltage as a function of time for two full cycles. c The corresponding X-ray attenuation (negative logarithm of X-ray transmission ${ }^{42}$ ) versus time. $\mathbf{d}$ Relative scattering intensity change as a function scattering vector length $q$ and time. e Ex situ SAXS/WAXS intensity versus scattering vector length $q$ of the empty activated carbon electrode and the same electrode filled with electrolyte. Nanopore (NP) scattering intensities, obtained by subtracting the particle scattering intensity and the electrolyte structure factor (Supplementary Fig. 8), are given in gray and light blue. The inset schematically shows the electron (scattering length) density contrast between carbon skeleton and pore. f SAXS/WAXS intensities versus scattering vector length $q$ during positive polarization. Color changes from red to blue with increasing cell voltage. The inset schematically shows electron (scattering length) density contrasts between carbon skeleton, electrolyte-filled pore and solid (crystalline) iodine. The scattering length densities of iodine, carbon and electrolyte are assumed with $3.49 \times 10^{11} \mathrm{~cm}^{-2}, 1.61 \times 10^{11} \mathrm{~cm}^{-2}$, and $1.01 \times 10^{11} \mathrm{~cm}^{-2}$, respectively. $\mathbf{g}$ The corresponding WAXS intensities during positive polarization, after subtracting the electrolyte structure factor contribution at $\mathrm{O} \mathrm{V}$ cell voltage. $\mathrm{I}_{2}$ crystal diffraction peak positions are indicated, (111) at $25.1 \mathrm{~nm}^{-1},(200)$ at $29.0 \mathrm{~nm}^{-1}$, and (220) at $41.0 \mathrm{~nm}^{-1}$.

compounds accumulating in its characteristic nanopores. The electron densities $\rho$ of carbon skeleton and electrolyte-filled nanopores (insert in Fig. $2 \mathrm{f}$ ) point at the formation of solid iodine since dissolved polyiodides alone could not account for the necessary electron density increase.

In the WAXS regime $\left(q>6 \mathrm{~nm}^{-1}\right)$, distinct intensity peaks form around $7.5 \mathrm{~nm}^{-1}, 16 \mathrm{~nm}^{-1}$, and $21.5 \mathrm{~nm}^{-1}$ (Fig. $2 \mathrm{~g}$ ). To better visualize the peak formation, we subtracted the structure factor contribution of the $1 \mathrm{M} \mathrm{NaI}$ electrolyte (that is, the WAXS intensity at $0 \mathrm{~V}$ cell voltage). We attribute the peak around $7.5 \mathrm{~nm}^{-1}$ to $\mathrm{I}_{3}{ }^{-}-\mathrm{I}_{3}{ }^{-}$correlations of a highly concentrated triiodide solution in the cavities formed by nanoporous carbon and solid $\mathrm{I}_{2}$, justified by the mean distance between $\mathrm{I}_{3}{ }^{-}$species being in the order of $2 \pi / 7.5 \mathrm{~nm}^{-1}=$ $0.84 \mathrm{~nm}$. The peaks at $16 \mathrm{~nm}^{-1}$ and $21 \mathrm{~nm}^{-1}$ do not exactly match diffraction peaks of crystalline $\mathrm{I}_{2}$, indicating that solid $\mathrm{I}_{2}$ forms in a crystal structure slightly different from bulk $\mathrm{I}_{2}$, partially in an amorphous state and/or the confined nanocrystals are strongly distorted. The diffraction peak widths (FWHM), as obtained by Gaussian peak fitting (Supplementary Fig. 7), are located in the region of $10.5^{\circ}-19^{\circ} 2 \theta$. According to Scherrer's equation, this translates into a crystallite size between $0.55-0.9 \mathrm{~nm}$, which fits well to the mean carbon pore size of $0.81 \mathrm{~nm}$ and the intensity changes found in SAXS.

Qualitatively, the in situ SAXS/WAXS and Raman data analysis provide correlating complementary information. Based on these data, we see that during oxidation, solid iodine particles are deposited in the carbon nanopores. At the same time, significant amounts of $\mathrm{I}_{3}{ }^{-}$and $\mathrm{I}_{5}{ }^{-}$are generated. The WAXS correlation peak at $7.5 \mathrm{~nm}^{-1}$ suggests a highly concentrated solution of polyiodides that is, to a large extent, confined to the nanopores. Solid iodine domains are likely to be present in crystalline form, since diffraction peak widths point at crystallite sizes up to $0.9 \mathrm{~nm}$, in accord with the mean carbon pore size of $0.81 \mathrm{~nm}$.

Quantification of iodine phase evolution via stochastic modeling. To quantify the amount of the formed $\mathrm{I}_{2}$ in the nanopores, we first separate the electrolyte/carbon structure factor (high q) and the particle scattering (low q) from the pure nanopore scattering (intermediate $q$ ). The separation procedure is described in the Methods and Supplementary Fig. 8. In a second step, we use the concept of plurigaussian random fields to fit the reduced experimental in situ SAXS curves and generate 3D lattice models of the carbon nanopore structure filled with the solid iodine phase. This involves modeling the empty carbon nanopore structure using a Gaussian random field (GRF) $Y(\mathbf{x})$ and the solid $\mathrm{I}_{2}$ via a second Gaussian random field $Z(\mathbf{x})$. After the carbon nanopore structure has been obtained from a fit to the SAXS intensity of the empty carbon electrode (Supplementary Fig. 9 and ref. ${ }^{45}$ ), the in situ SAXS data are fitted using the $I_{2}$ pore occupancy and two structural parameters of the $\mathrm{I}_{2}$ phase as fit 
parameters. These are the correlation parameter $l_{Z}$ of the GRF $Z$ $(\mathbf{x})$ and the parameter $\delta$ accounting for carbon-iodine correlations (Supplementary Fig. 10). A detailed description of the plurigaussian random field modeling and fitting is given in the Methods, limitations and sources of error in Supplementary Note 1.

The modeled in situ SAXS intensities fit well to the reduced experimental in situ SAXS intensities measured during positive polarization (Fig. 3a, b, $\mathrm{I}^{-}$oxidation proceeds from red to blue). The increasing scattering intensity is caused by the increasing amount of solid iodine as visualized on cross-sections and 3D cutouts in Fig. 3d (see Supplementary Video of the 3D model at maximum pore filling). The overall shape of the scattering curve remains similar to the shape of the empty nanopore structure (Fig. 2e and bottom red curve in Fig. 3a at the beginning of $\mathrm{I}^{-}$ oxidation). This finding is attributed to the similar characteristic feature size of the solid iodine structure and the empty carbon nanopores on the one hand (compare fit parameters $l_{Z}$ and $l_{Y}$, Supplementary Fig. 11), and the correlation between the iodine and carbon structure on the other hand (fit parameter $\delta$ ). Given the size of the confining carbon pore structure and the crystallite sizes obtained from the Scherrer analysis (Supplementary Fig. 7), the iodine particles should not be much larger than the mean pore size of $0.81 \mathrm{~nm}$. The $\mathrm{I}_{2}$ pore occupancy reaches a maximum of $30 \%$ at the maximum positive cell voltage of $0.8 \mathrm{~V}$ (Fig. 3c). This $\mathrm{I}_{2}$ pore occupancy fits well with the value estimated from
Faradaic capacity, assuming that all capacity forms $\mathrm{I}_{2}$ (blue line; in reality, a certain fraction also reacts to polyiodides). The SAXS integrated intensity analysis in Supplementary Note 2 provides further (model-free) evidence that the $\mathrm{I}_{2}$ pore occupancies shown in Fig. $3 \mathrm{c}$ lie in the right range. Given the poor electronic conductivity of $\mathrm{I}_{2}$, the found high degrees of pore filling are remarkable. We conclude that solid $\mathrm{I}_{2}$ rather than dissolved polyiodides represent the primary, capacity-relevant fraction of oxidized species. While in situ Raman data confirms the existence of significant amounts of $\mathrm{I}_{3}{ }^{-}$and $\mathrm{I}_{5}{ }^{-}$, relative to solid $\mathrm{I}_{2}$, their absolute amount is small at least up to charging capacities of $200 \mathrm{mAh} \mathrm{g}_{\mathrm{C}}^{-1}$.

The quantification via plurigaussian random fields, presented here, confirms the qualitative data interpretation from above. Contrary to the current state-of-the-art, oxidation of iodide in nanoporous carbon electrodes results in solid iodine nanoparticles, as well as dissolved $\mathrm{I}_{3}{ }^{-}$and $\mathrm{I}_{5}{ }^{-}$polyiodides. The physico-chemical mechanism to stabilize solid $\mathrm{I}_{2}$ is further discussed in Supplementary Note 3. A hysteresis in the iodine formation/dissolution as a function of the WE potential during charge/discharge suggests that solid $\mathrm{I}_{2}$ deposited in the porous carbon requires a significant overpotential to be dissolved (Supplementary Note 3). This is in line with the stabilization of solid $\mathrm{I}_{2}$ by severe carbon-iodine interactions in the nanoporous confinement.

Processes in the positive electrode of a hybrid NaI supercapacitor are comparable to those in common conversion-type
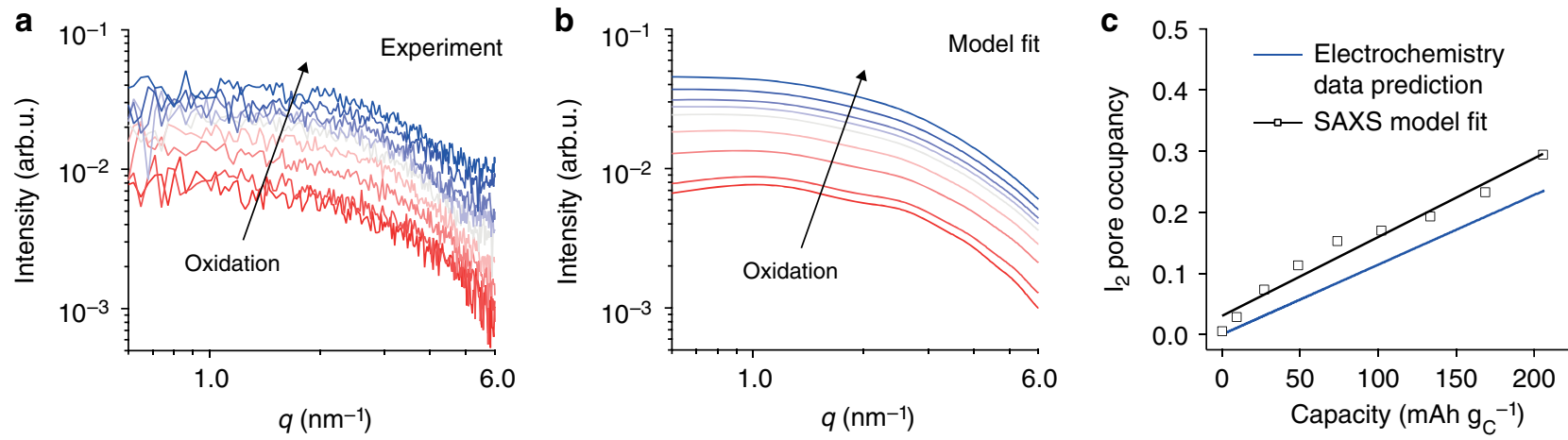

\section{d}

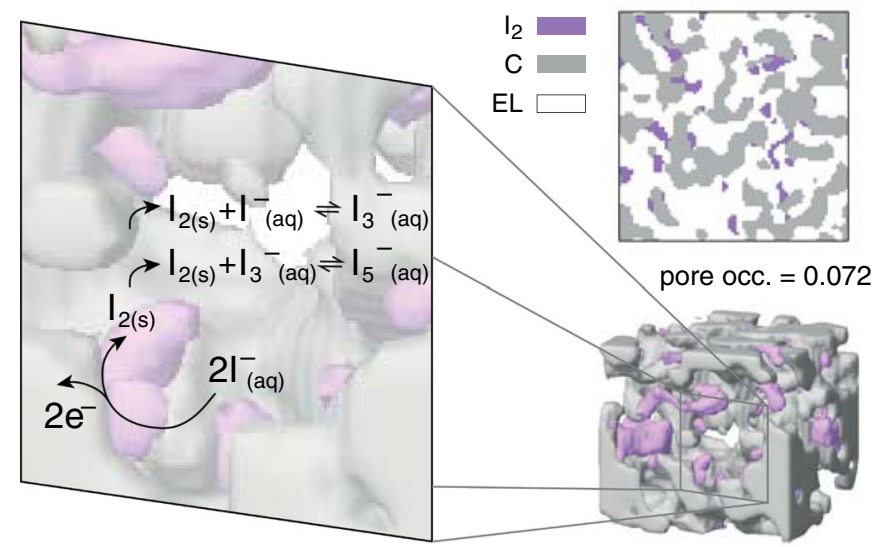

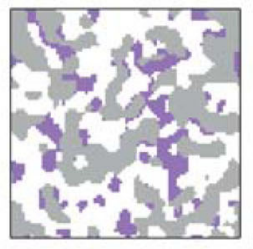

pore occ. $=0.172$

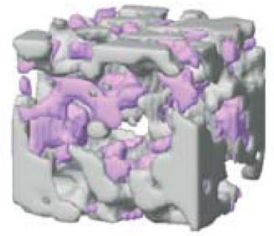

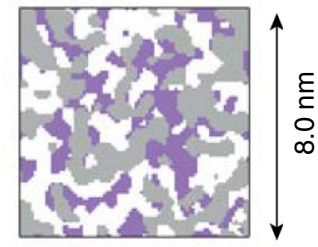

pore occ. $=0.295$

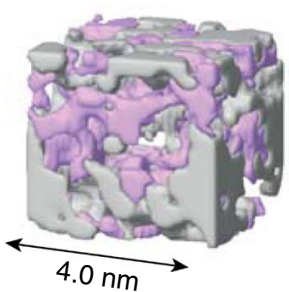

Fig. 3 Reaction mechanism of $\mathbf{I}_{\mathbf{2}}$ formation and quantification of phase evolution via stochastic modeling. a Reduced experimental in situ SAXS data during positive cell polarization (after subtraction of particle and electrolyte structure factor scattering contributions, see Supplementary Fig. 8). b Best-fit modeled small-angle scattering intensities versus scattering vector length $q$ for fractions for an increasing fraction of iodine phase (from red to blue curves), using the concept of plurigaussian random fields (see Methods). c lodine pore occupancy vs. specific capacity as obtained from the in situ SAXS data fits (black, solid line represents linear fit) and estimated from specific capacity and known electrode porosity. d 3D lattice models generated by plurigaussian random fields for increasing iodine pore occupancies (pore occ.). The cross-sections (top) and 3D cut-outs correspond to the modeled in situ SAXS data shown in $\mathbf{b}$. The reaction mechanism derived from in situ Raman and in situ SAXS/WAXS data is shown schematically in the detail on the left. 
battery electrodes, such as Li-S or Li- $\mathrm{O}_{2}$ battery cathodes, where solid $\mathrm{Li}_{2} \mathrm{~S}$ or $\mathrm{Li}_{2} \mathrm{O}_{2}$ precipitate from solution species ${ }^{46,47}$. During charging and discharging, the solid active material is deposited within the nanopore network of a carbon cathode. The pore occupancy of active material hence determines achievable capacities and reversibility.

The iodide formation reaction mechanism. The in situ and ex situ data allow deriving a detailed reaction mechanism of the (poly)iodide/iodine redox chemistry in nanopore confinement (Fig. 3d). Specifically, (i) in situ Raman, ex situ UV-vis and in situ SAXS (Figs. 1-3) confirm the formation of $\mathrm{I}_{2}, \mathrm{I}_{3}{ }^{-}$and $\mathrm{I}_{5}{ }^{-}$during $\mathrm{I}^{-}$oxidation. (ii), $\mathrm{I}_{5}{ }^{-}$generation is delayed with respect to $\mathrm{I}_{3}{ }^{-}$ upon oxidation process as seen in their Raman intensities (Fig. 1f-g). This indicates that larger amounts of generated $\mathrm{I}_{3}{ }^{-}$ and $\mathrm{I}_{2}$ accelerate $\mathrm{I}_{5}{ }^{-}$formation. (iii), In situ SAXS/WAXS data confirm the formation of solid $\mathrm{I}_{2}$ nanoparticles or clusters in the nanopores.

At first, $\mathrm{I}^{-}$is oxidized to $\mathrm{I}_{2}$ at the carbon-electrolyte interface (Eq. (1)). This reaction leads to solid $I_{2}$ nanoparticles with a size limited by the electron tunneling/conduction thickness of the insulating $\mathrm{I}_{2}$ and the confining carbon cavities. Concurrently, $\mathrm{I}_{2}$ comproportionates to some extent with $\mathrm{I}^{-}$to $\mathrm{I}_{3}{ }^{-}$(Eq. (2)), with the amount of the latter growing with the amount of $\mathrm{I}_{2}$ (steadily increasing amounts of $\mathrm{I}_{3}{ }^{-}$and $\mathrm{I}_{2}$, Figs. 1 and 3). As $\mathrm{I}_{3}{ }^{-}$and $\mathrm{I}_{2}$ amounts grow in the nanopores, the $\mathrm{I}_{5}{ }^{-}$formation via the comproportionation (Eq. (3)) accelerates.

$$
\begin{aligned}
& 2 \mathrm{I}_{(\mathrm{aq})}^{-} \rightarrow \mathrm{I}_{2(\mathrm{~s})}+2 \mathrm{e}^{-} \\
& \mathrm{I}_{2(\mathrm{~s})}+\mathrm{I}_{(\mathrm{aq})}^{-} \rightleftharpoons \mathrm{I}_{3(\mathrm{aq})}^{-} \\
& \mathrm{I}_{2(\mathrm{~s})}+\mathrm{I}_{3(\mathrm{aq})}^{-} \rightleftharpoons \mathrm{I}_{5(\mathrm{aq})}^{-}
\end{aligned}
$$

The direct electrochemical generation of $\mathrm{I}_{3}^{-}$via $3 \mathrm{I}^{-} \rightarrow \mathrm{I}_{3}^{-}+$ $2 \mathrm{e}^{-}$can be excluded since the further precipitation of $\mathrm{I}_{2}$ via Eq. (2) would require to first reach an $\mathrm{I}_{3}{ }^{-}$concentration which would drive disproportionation; hence, $\mathrm{I}_{3}{ }^{-}$would level off while $\mathrm{I}_{2}$ grows. Instead, in situ Raman and SAXS show steadily and concurrently evolving amounts of $\mathrm{I}_{3}{ }^{-}$and $\mathrm{I}_{2}$. Based on the equilibrium constant of Eq. (2), the concentration of $\mathrm{I}_{3}{ }^{-}$is orders of magnitudes higher than the $I_{2}$ concentration at all times ${ }^{40,41,48}$. The generation of large amounts of $\mathrm{I}_{2}$ via precipitation (Eq. (2)) would require extremely high $\mathrm{I}_{3}-$ concentrations, which appears unlikely.

Low self-discharge, high capacity density iodine energy storage. The detected amount of solid $\mathrm{I}_{2}$ produced in the carbon nanopores during voltage cycling is quite remarkable, with a specific capacity reaching $200 \mathrm{mAh} \mathrm{g}_{\mathrm{C}}{ }^{-1}$. To gauge the maximum amount of iodine possible to be electrodeposited in the positive AC electrode, neither the negative AC electrode capacitance nor total $\mathrm{I}^{-}$in the system must limit iodine electrodeposition. This is realized with a large, about 100 times oversized AC counter electrode and a large electrolyte volume (details in Methods, cyclic voltammetry in Supplementary Fig. 12).

The system shows an excellent rate performance as shown by galvanostatic charge/discharge measurements with a set capacity limit of $160 \mathrm{mAh} \mathrm{g}_{\mathrm{C}}^{-1}$ (Fig. 4a, b). Note that the capacity limit rather than the potential limit needs to be set for this system, since polyiodide shuttling between WE and CE at high capacities hinders a rise in the WE potential when all pores are occupied by $\mathrm{I}_{2}$. The cycle life is very high and the Coulombic efficiency is constantly close to $100 \%$ (Fig. 4c). There are no signs for performance degradation after cycling several hundred times. Both rate capability and cycle life are comparable to hybrid supercapacitors even though the stored energy is much higher.
To check for the maximum capacity that can be practically used in the positive AC electrode, we oxidized $\mathrm{I}^{-}$at a constant potential of $+0.6 \mathrm{~V}$ vs $\mathrm{Ag} / \mathrm{AgCl}$ at the positive working electrode up to a certain charging capacity. After leaving the cell at opencircuit for different resting times (1-32 h), the remaining $\mathrm{I}_{2}$ was measured by a potentiostatic discharge to $0 \mathrm{~V}$ for $4 \mathrm{~h}$ (Fig. $4 \mathrm{~d}$ ).

The self-discharge increases as the charging capacity of the positive AC electrode is increased (Fig. 4e and Supplementary Fig. 13). Independent of the positive electrode loading, already after a few hours resting time, the discharge capacities approached a similar level. This is also shown by charging to three different capacities $\left(219 \mathrm{mAh} \mathrm{g}_{\mathrm{C}}^{-1}, 330 \mathrm{mAh} \mathrm{g}_{\mathrm{C}}{ }^{-1}\right.$, and $441 \mathrm{mAh} \mathrm{g}_{\mathrm{C}}^{-1}$ ) and waiting for $4 \mathrm{~h}$ at OCV (Supplementary Fig. 13). After $32 \mathrm{~h}$ of resting, the discharge capacities reached a value of $150 \mathrm{mAh} \mathrm{g}_{\mathrm{C}}{ }^{-1}$. Assuming that most capacity is stored in the form of solid $\mathrm{I}_{2}$ and by considering the known electrode porosities (Supplementary Table 1) this corresponds to an $I_{2}$ pore occupancy of 0.17 .

The proposed reaction mechanism explains the increasing selfdischarge with increasing amounts of iodine loading/charging capacities. The more iodine is deposited, the more mobile $\mathrm{I}_{3}^{-}$and $\mathrm{I}_{5}{ }^{-}$are formed chemically. Owing to their negative charge, a certain fraction of polyiodides will remain adsorbed in the nanopores of the positively polarized WE. The more polyiodides are generated, the higher is also their fraction that diffuses to the $\mathrm{CE}$, where they are reduced to $\mathrm{I}^{-}$to promote self-discharge. Hence, future strategies to increase the iodine pore occupancy and reduce self-discharge should focus on reducing the concentration of shuttling polyiodides. Based on the reaction mechanism in Fig. 3d (Eqs. (2) and (3)), this involves reducing the $\mathrm{I}^{-}$concentration by, for example, using additional, electrochemically inert anions like $\mathrm{NO}_{3}{ }^{-}$, which allow precipitating a larger fraction of the $\mathrm{I}^{-}$without anion starvation. Using aqueous $0.5 \mathrm{M} \mathrm{NaI}$ and $0.5 \mathrm{M} \mathrm{NaNO}_{3}$ as electrolyte (red curve, Fig. $4 \mathrm{e}$ ), $\mathrm{I}_{2}$ pore filling and discharge capacities improve significantly. This not only demonstrates a practical way towards improved energy densities of carbon-iodine electrodes used in hybrid supercapacitors or batteries, but also independently confirms the reaction mechanism, derived from in situ Raman and SAXS/ WAXS.

\section{Discussion}

In situ Raman and in situ SAXS/WAXS have shown that oxidation of $\mathrm{I}^{-}$in the confinement of carbon nanopores generates not only $\mathrm{I}_{3}^{-}$and $\mathrm{I}_{5}^{-}$polyiodides, but to a large extend solid iodine nanoparticles. Induced by the confinement of the carbon nanopores, solid $\mathrm{I}_{2}$ dissolves only slowly during self-discharge. The derived reaction mechanism explains all recorded in situ spectroscopic, in situ scattering, and electrochemical data and suggests pathways to increase capacity densities and further reduce selfdischarge. Electrochemical measurements evidence remarkably high capacities. In terms of specific and volumetric capacity solid $\mathrm{I}_{2}\left(211 \mathrm{mAh} \mathrm{g}^{-1}, 1040 \mathrm{mAh} \mathrm{cm}^{-3}\right)$ surpasses todays benchmark Li-ion cathode materials, such as $\mathrm{LiFePO}_{4}\left(170 \mathrm{mAh} \mathrm{g}{ }^{-1}\right.$, $596 \mathrm{mAh} \mathrm{cm}^{-3}$ ). To make capacity values comparable to intercalation-type cathode materials the total capacity of the iodine system needs to be normalized by the mass or volume of carbon, micropore electrolyte and deposited $\mathrm{I}_{2}$. An $\mathrm{I}_{2}$ pore occupancy of $40 \%$ in our carbon with $60 \%$ porosity would result in practical capacities of $108.5 \mathrm{mAh} \mathrm{g}^{-1}$ and $250 \mathrm{mAh} \mathrm{cm}^{-3}$. We show how to achieve such high packing densities at rates significantly exceeding those of Li-ion cathode materials. The absence of slow intercalation processes enables rates comparable to hybrid supercapacitors. An effective strategy to improve the iodine pore filling capacities lies in reducing the concentration of 

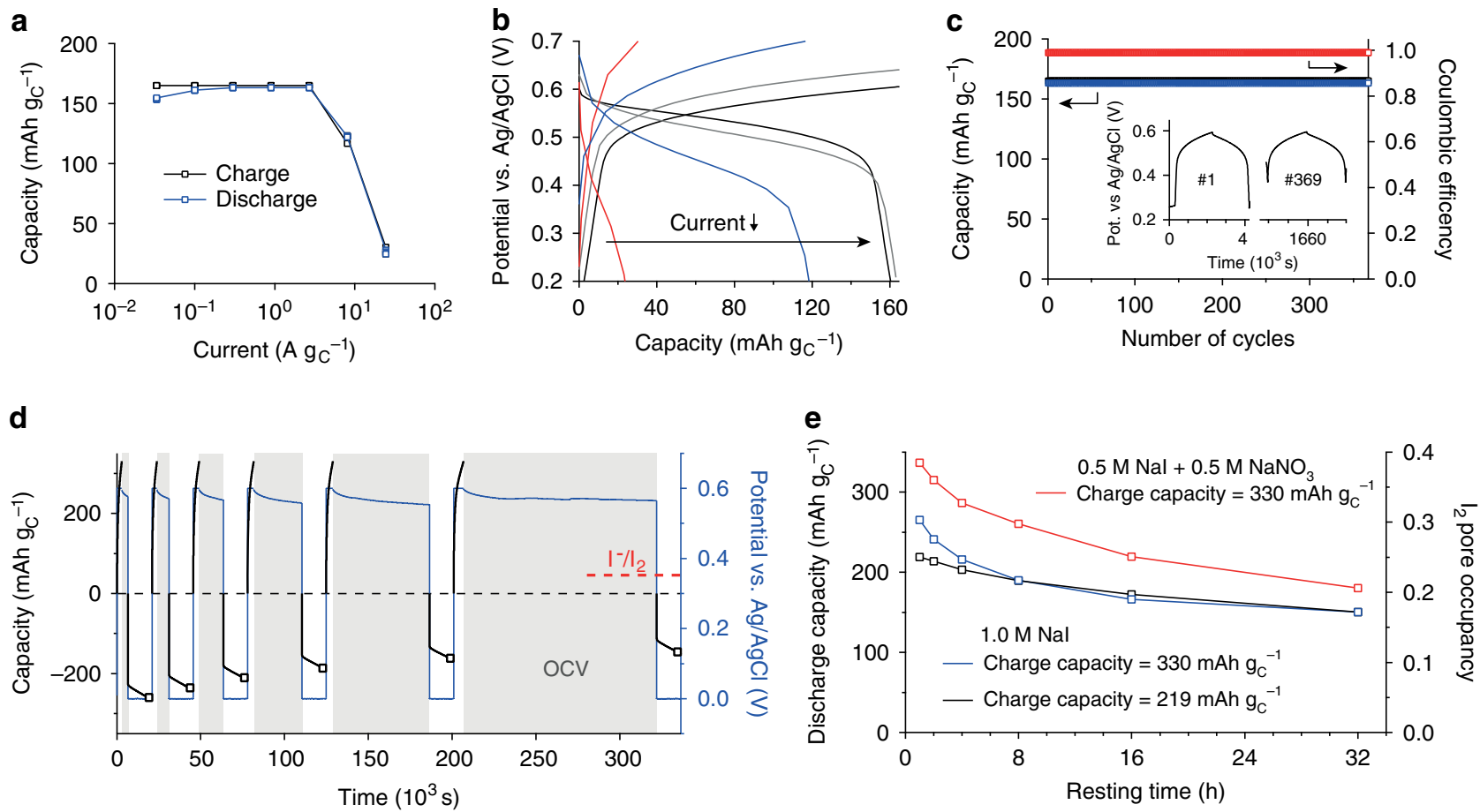

Fig. 4 Low self-discharge, high capacity density iodine energy storage. a Specific capacity versus specific current for galvanostatic charge/discharge with capacity limitation of $160 \mathrm{mAh} \mathrm{gc}^{-1}$. b WE potential as a function of capacity for the four highest currents with $24.3 \mathrm{~A} \mathrm{~g}^{-1}$ (red), $8.1 \mathrm{~A} \mathrm{gc}^{-1}$ (blue), $2.7 \mathrm{~A} \mathrm{gc}_{\mathrm{c}}^{-1}$ (gray), $0.9 \mathrm{~A} \mathrm{gc}_{\mathrm{c}}^{-1}$ (black) of the same galvanostatic charge/discharge measurements. c Charge/discharge capacity and coulombic efficiency versus number of cycles at a rate of $0.3 \mathrm{AgC}^{-1}$. The inset shows the WE potential as a function time for 2 cycles. $\mathbf{d}$ Potentiostatic charge / discharge $(+0.6 / 0 \mathrm{~V}$ vs. $\mathrm{Ag} / \mathrm{AgCl})$ measurements with increasing resting times at open-circuit voltage $(\mathrm{OCV})$ between the charging and discharging steps. Potentiostatic charge was limited to $330 \mathrm{mAh} \mathrm{gC}^{-1}$, potentiostatic discharge to $4 \mathrm{~h}$ within which the current always dropped to negligible values. The protocol is exemplified for the $1 \mathrm{M} \mathrm{Nal}$ electrolyte and a charging capacity of $330 \mathrm{mAh} \mathrm{gC}_{\mathrm{C}}{ }^{-1}$. e Discharge capacities vs. resting times for the protocol shown in a using the $1 \mathrm{M} \mathrm{Nal}$ electrolyte and two different charging capacities ( $219 \mathrm{mAh} \mathrm{gc}^{-1}$ and $330 \mathrm{mAh} \mathrm{gc}_{\mathrm{c}}^{-1}$ ) and the $0.5 \mathrm{M} \mathrm{Nal}+0.5 \mathrm{M} \mathrm{NaNO}$ electrolyte

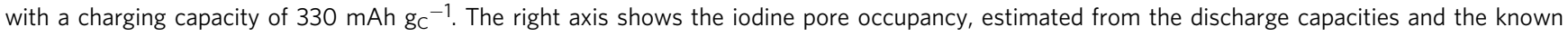
electrode porosity values.

iodide in the carbon nanopores in the charged state using an inert supporting electrolyte. Further performance increase relies on systematic parameter studies varying the nanopore structure of the used carbons, the electrolyte volume, salt concentration, and the amount of supporting electrolyte.

Understanding that $\mathrm{I}^{-}$oxidation in carbon nanopores electrodeposits solid iodine calls for a paradigm shift in two aspects. First, increasing the capacity of such systems relies on strategies to increase the pore filling with solid iodine. Second, avoiding self-discharge means reducing the concentration of mobile polyiodide species generated by comproportionation. Until now, confined immobile polyiodides were thought to be responsible for the excellent self-discharge properties of iodine-based energy storage.

Considering the shown strategies to improve capacity and selfdischarge, the similarities with other conversion-type batteries, such as Lithium-sulfur, are remarkable. Carbon electrodes with a high degree of iodine pore filling are thus alternative, sustainable, and environmentally friendly conversion-type battery electrodes that may be used in combination with a supercapacitor counterelectrode (hybrid supercapacitor) or a battery anode material (e.g., zinc-iodine battery).

From a broader perspective, the work shows the necessity of applying advanced in situ techniques for detailed insights into electrochemical mechanisms and to derive practical conclusions for real devices. We show that properties and function of complex emerging systems for electrochemical energy storage are equally controlled by chemistry (in situ spectroscopy) and the nanoscale structure (in situ scattering). In combination with stochastic modeling, in situ SAXS/WAXS allowed for quantifying the iodine pore filling and visualizing the (sub-)nm iodine phase evolution during electrochemical cycling. Hence, in situ SAXS proves as a powerful experimental method to study the phase evolution of active materials in nanoporous carbons and beyond.

\section{Methods}

Materials. Free-standing carbon electrodes were prepared by mixing 90 mass $\%$ of the microporous $\mathrm{KOH}$-activated carbon (MSP-20, Kansai Coke and Chemicals, Supplementary Fig. 1, Supplementary Table 1) with 5 mass\% carbon black (C65 from Imerys), and 5 mass $\%$ of polytetrafluoroethylene (60\% dispersion in water from $3 \mathrm{M}$ Chemicals) in ethanol. After stirring at $+70^{\circ} \mathrm{C}$, the obtained dough was pressed and rolled on a glass plate into a thin sheet. Disc electrodes were punched from the sheet and dried at $120^{\circ} \mathrm{C}$, resulting in a final thickness of about $150 \mu \mathrm{m}$. Sodium iodide (NaI, 99.5\%) was purchased from Alfa-Aesar and dried at $110^{\circ} \mathrm{C}$ overnight before preparing the aqueous $1.0 \mathrm{M} \mathrm{NaI}$ electrolyte with de-ionized water. The $\mathrm{pH}$ of the electrolyte was 6.5 and the conductivity $82 \mathrm{mS} \mathrm{cm}^{-1}$.

Raman spectroscopy. In situ Raman spectroscopy was carried out using a LabRAM HR 800 spectrometer combined with an Olympus BX41 microscope. The spectra were measured from the top, with the focus plane underneath the standard

microscope glass slip (covering the cell assembly) and the electrolyte film on top of the carbon electrode. A schematic drawing of the two-electrode electrochemical in situ cell ECC-Opto-Gas from EL-CELL (Hamburg, Germany) is given in Supplementary Fig. 2. As an objective lens a 40× Olympus LUCPlanFL N $(\mathrm{NA}=0.6$; corrected for the cover thickness) was used. To avoid sample damage, the laser with $532 \mathrm{~nm}$ wavelength worked at reduced power $(0.5 \mathrm{~mW})$. With the given grating/slit setup $\left(300 \mathrm{~mm}^{-1} ; 200 \mu \mathrm{m}\right)$ the spectral (pixel) resolution is about $3.6 \mathrm{~cm}^{-1}$. The acquisition time per spectrum was $30 \mathrm{~s}$ (4-fold accumulations) for a total measurement time and time resolution of $120 \mathrm{~s} / \mathrm{spectrum}$. In addition, the DuoScan System was applied to continuously scan the laser spot over a $20 \times 20 \mu \mathrm{m}$ area. With 
regard to the spectral analysis the band deconvolution of the $\mathrm{I}_{3}{ }^{-} / \mathrm{I}_{5}{ }^{-}$bands (Supplementary Fig. 3) was done using three Lorentz peaks (initial position $110 \mathrm{~cm}^{-1}$ $143 \mathrm{~cm}^{-1}$, and $224 \mathrm{~cm}^{-1}$ ) and one Gaussian peak (initial position $165 \mathrm{~cm}^{-1}$ ), this routine is based on the band assignments from ref. ${ }^{27}$. The deconvolution of the G- and D-band was done according to the method introduced in ref. ${ }^{34}$.

UV-vis spectroscopy. Positive carbon electrodes (mass $=3.0 \mathrm{mg}$ ) were charged in a two-electrode cell with oversized counter-electrode (mass $=34.0 \mathrm{mg}$ ) by voltage sweep $\left(2.0 \mathrm{mV} \mathrm{s}^{-1}\right)$ to a cell voltage of $1.0 \mathrm{~V}$. After charging, the positive electrodes were taken out, washed for $1 \mathrm{~min}$ with deionized water and placed for $3 \mathrm{~h}$ in three different solutions: (i) deionized water, (ii) aqueous $1 \mathrm{M} \mathrm{NaI}$ and (iii) ethanol. UVVis absorption spectra of the solutions were recorded on a Cary 50 spectrophotometer (Varian).

Small and wide-angle X-ray scattering. In situ SAXS/WAXS measurements were carried out with a custom-built two-electrode electrochemical in situ cell. The cell consisted of the positive AC electrode, a glass fiber separator (Whatman), a negative AC electrode and platinum foil current collector (CC). The assembly was sealed in a stainless-steel housing with adhesive film (Tixo) X-ray windows. To enhance the recorded signal by increasing the amount of oxidized $\mathrm{I}^{-}$the negative electrode was oversized 8-times (mass $=32 \mathrm{mg}$, thickness $=600 \mu \mathrm{m}$, diameter $=$ $12 \mathrm{~mm}$ ) compared to the positive electrode (mass $=4 \mathrm{mg}$, thickness $=200 \mu \mathrm{m}$, diameter $=8 \mathrm{~mm}$ ). Oversizing the negative electrode requires increasing its thickness rather than increasing the diameter to avoid detrimental, large ion diffusion pathways. To investigate only processes in the positive electrode, a hole with $2 \mathrm{~mm}$ diameter was cut into negative electrode, separator and CC.

SAXS/WAXS data were recorded on a laboratory SAXS/WAXS facility (SAXSpoint 2.0, Anton Paar, Graz, Austria) using $\mathrm{Cu}-\mathrm{Ka}$ radiation and a 2D areal detector (Eiger R 1M, Dectris, Baden, Switzerland) with a nominal sample-todetector distance of $100 \mathrm{~mm}$. The primary 2D scattering data were azimuthally averaged and normalized by transmission values and recording time. Further, intensity data were multiplied by polarization $2 /\left(1+(\cos 2 \theta)^{2}\right)$ and absorption $\left[1-\tau^{\frac{1}{\cos 2 \theta}-1}\right] /\left[\ln \tau-\frac{1}{\cos 2 \theta} \ln \tau\right]$ correction factors to adequately normalize scattering intensities at large scattering angles $2 \theta^{49}$. The background scattering intensity was recorded after the in situ SAXS measurements and removing the WE; and subtracted from in situ SAXS/WAXS data after primary data treatment as described above.

To separate the nanopore scattering contribution from the electrolyte structure factor and the low- $q$ particle scattering, we chose a pragmatic approach: we first determined the electrolyte structure factor using a modified Porod fit of the form $I_{\text {porod }}=P / q^{4}+A \cdot S F(q)$ at $6.5 \mathrm{~nm}^{-1}<q<9 \mathrm{~nm}^{-1}$, where $S F(q)$ is the electrolyte structure factor, $A$ a multiplicative constant, and $P$ the Porod constant. We assume that $S F(q)$ corresponds approximately to the measured scattering intensity of the bulk electrolyte (as shown in Supplementary Fig. 8) and remains constant during cycling the cell. The parameters $A$ and $P$ were determined by the fit to the electrolyte filled AC electrode at zero cell voltage. After subtracting the electrolyte structure factor contribution $A \cdot S F(q)$, the particle scattering contribution was obtained from a power law fit of the form $\mathrm{I}_{\text {particle }}=B / q^{3.73}+C$ at $0.4 \mathrm{~nm}^{-1}<q<$ $1 \mathrm{~nm}^{-1}$, with $B$ and $C$ being the unknown fit parameters. The exponent of 3.73 was determined from a power law fit to the empty (bare) AC electrode. While $A \cdot S F(q)$ is assumed to be constant, the power law fit at low $q$ with changing parameters $B$ and $C$ was carried out for all in situ SAXS intensities during cell polarization. The obtained particle scattering $B / q^{3.73}$ was then subtracted from the experimental SAXS intensities.

The in situ SAXS data fits apply the concept of plurigaussian random fields ${ }^{45}$, as explained below. To ensure proportionality between experimental and modeled SAXS intensities we multiplied the modeled SAXS intensities by a factor $K$, given in Eq. (4). The constant $K$ was determined once, by dividing the integrated intensity $\tilde{I}=\int_{0}^{\infty} q^{2} I(q) d q$ of the experimental nanopore scattering intensity (with no iodine present) by the integrated intensity of the corresponding modeled SAXS intensity 45,50 . We assume the carbon nanopore structure and the corresponding GRF $Y(\mathbf{x})$ to be constant during the in situ SAXS experiment. The GRF parameters of $Y(\mathbf{x})$ are taken from a fit to the experimental ex situ SAXS intensity of the empty nanoporous carbon electrode (Supplementary Fig. 9 and Supplementary Table 2). The nanopore scattering intensity of the bare AC electrode was obtained by subtracting a constant background (determined via a standard Porod fit in the $q$ range $7 \mathrm{~nm}^{-1}<q<9 \mathrm{~nm}^{-1}$ ) and the particle scattering contribution (determined by a power-law fit $I_{\text {power }}=D / q^{\eta}+E$, for $q<1 \mathrm{~nm}^{-1}$ ) from the SAXS intensity of the empty AC electrode (solid black line). Since the nanopore scattering intensity is fitted with a simple two-phase model (see below), density fluctuations within the carbon matrix ${ }^{51}$ are neglected. We assumed a porosity of $1-\phi_{C}=60 \%$ as obtained from gas sorption measurements (Supplementary Table 1), set the upper threshold value to $\infty$, and used $l_{Y}$ and $d_{Y}$ as fit parameters.

The in situ SAXS intensities (Fig. 3a) were fitted to three fit parameters: the $\mathrm{I}_{2}$ pore occupancy $\phi_{A}$, the correlation parameter $l_{Z}$ of the iodine phase GRF $(Z(\mathbf{x}))$, and the parameter $\delta$, accounting for $Z(\mathbf{x})$ (iodine) and $Y(\mathbf{x})$ (carbon) correlations. The $\mathrm{I}_{2}$ pore occupancy values were constrained to values smaller than 0.35 , estimated from electrochemical capacity values (Fig. 3c). The value $d_{Z}$ was kept constant at a value of $30 \mathrm{~nm}\left(d_{Z} \approx \infty\right)$. The scattering length densities of iodine, carbon and electrolyte were assumed with $3.49 \times 10^{11} \mathrm{~cm}^{-2}, 1.61 \times 10^{11} \mathrm{~cm}^{-2}$, and $1.01 \times 10^{11} \mathrm{~cm}^{-2}$, respectively. This corresponds to a mass density of $1.9 \mathrm{~g} \cdot \mathrm{cm}^{-3}$ for the carbon skeleton, following previous works ${ }^{43}$, and $4.94 \mathrm{~g} \mathrm{~cm}^{-3}$ for iodine, corresponding to the iodine crystal density. The scattering length density of the electrolyte corresponds to a concentration of $1 \mathrm{M} \mathrm{NaI}$ in the aqueous solvent. To fit the in situ SAXS intensities, we minimized the sum of the squared residuum of all experimental SAXS intensities during a positive potential sweep. In other words, the parameters $l_{Z}$ and $\delta$ were held constant while the iodine pore occupancy was used as fit parameter for each SAXS curve at its specific state of charge. The procedure was repeated for several values of $l_{Z}$ and $\delta$ (Supplementary Fig. 11) and the parameter set with the minimum sum of squared residuum values taken as the solution.

Plurigaussian random fields. We model the in situ SAXS data using the concept of plurigaussian random fields ${ }^{45}$. This allows retrieving $3 \mathrm{D}$ real space models of the solid iodine phase evolution within the carbon nanopore structure and quantifying the degree of iodine pore filling (Fig. 3). A detailed description of how plurigausian random fields are applied to in situ SAXS data of three-phase systems is given by Gommes et al. ${ }^{45}$

The SAXS intensity $I(q)$ as a function of the scattering vector length $q$ is the Fourier transform of the electron density correlation function $C(r)$

$$
I(q)=K \int_{0}^{\infty} C(r) \frac{\sin (q r)}{q r} 4 \pi r^{2} d r,
$$

with $K$ being a constant that depends on the sample volume and instrumental parameters, such as detector efficiency. $C(r)$ for a three-phase system consisting of phases $\mathrm{A}, \mathrm{B}, \mathrm{C}$ can be written as

$$
\begin{aligned}
C(r)= & \left(\rho_{\mathrm{A}}-\rho_{\mathrm{B}}\right)\left(\rho_{\mathrm{A}}-\rho_{\mathrm{C}}\right)\left[P_{\mathrm{AA}}(r)-\phi_{\mathrm{A}}^{2}\right] \\
& +\left(\rho_{\mathrm{B}}-\rho_{\mathrm{A}}\right)\left(\rho_{\mathrm{B}}-\rho_{\mathrm{C}}\right)\left[P_{\mathrm{BB}}(r)-\phi_{\mathrm{B}}^{2}\right] \\
& +\left(\rho_{\mathrm{C}}-\rho_{\mathrm{A}}\right)\left(\rho_{\mathrm{C}}-\rho_{\mathrm{B}}\right)\left[P_{\mathrm{CC}}(r)-\phi_{\mathrm{C}}^{2}\right] .
\end{aligned}
$$

Here, $\rho_{\mathrm{i}}$ is the electron density, $\phi_{\mathrm{i}}$ the volume fraction and $P_{\mathrm{ii}}(r)$ the two-point correlation function of phase $\mathrm{i}$.

Before modeling the iodine phase evolution, we generate a 3D model of the empty nanoporous carbon structure using a fit to the ex situ SAXS curve of the empty carbon electrode and the concept of clipped Gaussian random fields. Detailed descriptions of the procedure are given in refs. $31,43,50,52$. In brief, a Gaussian random field $Y(\boldsymbol{x})$ is the sum of cosine waves with wave vectors distributed according to their power spectral density $f_{Y}(k)$ and phase factors $\varphi_{\mathrm{i}}$ randomly distributed between 0 and $2 \pi^{45,50,53,54}$.

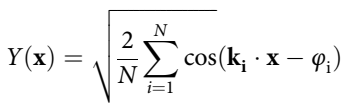

A suitable analytic two-point correlation function of the GRF is ref. ${ }^{31}$

$$
g_{Y}(r)=\frac{1}{\cosh \left(r / l_{Y}\right)} \frac{\sin \left(2 \pi r / d_{Y}\right)}{\left(2 \pi r / d_{Y}\right)},
$$

where $l_{Y}$ is a correlation parameter related to the mean size of the pores and $d_{Y}$ a parameter accounting for ordering effects in-between pores. Equation S4 translates into the following analytic expression for the power spectral density:

$$
f_{Y}(k)=\frac{k}{\pi} l_{Y} d_{Y} \frac{\sinh \left(\pi k l_{Y} / 2\right) \sinh \left(\pi^{2} l_{Y} / d_{Y}\right)}{\cosh \left(\pi k l_{Y}\right)+\cosh \left(2 \pi^{2} l_{Y} / d_{Y}\right)} .
$$

To generate a two-phase porous structure from the GRF, we define threshold values $\alpha$ and $\beta$ for the Gaussian distributed $Y(\boldsymbol{x})$ values. All spatial coordinates $\boldsymbol{x}$ with $\alpha<Y(\boldsymbol{x}) \leq \infty$ are assigned to the pore space; all other coordinates to the carbon skeleton. The two threshold values are related to the carbon volume fraction $\phi_{\mathrm{C}}$ via:

$$
\phi_{\mathrm{C}}=\frac{1}{\sqrt{2 \pi}} \int_{\alpha}^{\infty} \exp \left(-\frac{t^{2}}{2}\right) d t .
$$

To generate the SAXS intensity from the two-phase pore structure we calculate the two-point probability function $P_{\mathrm{CC}}(r)$ via

$$
P_{\mathrm{CC}}(r)=\frac{1}{2 \pi} \int_{0}^{g_{\mathrm{Y}}(r)} \frac{1}{\sqrt{1-t^{2}}} \exp \left(-\frac{\alpha^{2}}{1+t}\right) d t+\phi_{\mathrm{C}}^{2}
$$

The SAXS intensity of the empty carbon nanopore structure is generated by inserting $P_{\mathrm{CC}}(r)$ in Eqs. (4) and (5) (Supplementary Fig. 9, Supplementary Table 2). Note that in previous papers the structure of the same activated carbon was modeled by intersecting two independently generated GRFs. Based on the plurigaussian random field approach applied in this work we do not use intersected GRF structures.

To model SAXS intensities and real space structures of the solid iodine phase (phase A) within the nanopores, we generate a second independent GRF $Z(\mathbf{x})$ using the correlation function in Eqs. (7) and (8) with different input parameters $l_{Z}$ and $d_{Z}$ (Supplementary Fig. 10c). We set $d_{Z} \rightarrow \infty$, i.e. the oscillation factor in Eq. (7) becomes 1 and $g_{Z}(r)=1 / \cosh \left(r / l_{Z}\right)$. Phase A with the volume fraction $\phi_{\mathrm{A}}$ within the pore structure is generated by cutting $Z(\mathbf{x})$ and $Y(\mathbf{x})$ according to Eq. (11) and 
the cut-offs visualized in Supplementary Fig. 10d-f.

$$
\phi_{\mathrm{A}}=\iint_{(Y, Z) \in D_{\mathrm{A}}} \frac{1}{2 \pi} \exp \left(-\frac{Y^{2}+Z^{2}}{2}\right) d Y d Z
$$

The two-point correlation functions of phase A (and B) are calculated via

$$
P_{\mathrm{AA}}=\int_{D_{\mathrm{A}}} d Y_{1} d Z_{1} \int_{D_{\mathrm{A}}} d Y_{2} d Z_{2} G_{g_{Y}(r)}\left(Y_{1}, Y_{2}\right) G_{g_{Z}(r)}\left(Z_{1}, Z_{2}\right)
$$

with $G_{g}\left(Y_{1}, Y_{2}\right)$ being the bivariate Gaussian distribution with mean 0 , variance 1, and covariance $g$. Their calculation via Hermite polynomials is described in ref. ${ }^{45}$. Depending on the angle $\delta$ and the A/B boundary line in Supplementary Fig. 10d-f the morphology of phase A will be different. Phase A will perfectly cover/wet the carbon phase in form of a thin film if $\delta \rightarrow 0$ (Supplementary Fig. 10d, g). In contrast, for an $\mathrm{A} / \mathrm{B}$ boundary parallel to the $\mathrm{Y}$-axis $(\delta \rightarrow \pi / 2)$ the structures of phase $\mathrm{A}(\mathrm{B})$ are statistically independent from phase C (Supplementary Fig. 10f, i). Inserting Eq. (12) into Eqs. (4) and (5) gives the corresponding scattering intensities (Supplementary Fig. 11). Sources of error and limitations of the plurigaussian model fit are discussed in Supplementary Note 1.

Electrochemical characterization. Three-electrode Swagelok-type cells with a Ag/ $\mathrm{AgCl}$ reference electrode were assembled for cyclic voltammetry (Supplementary Fig. 12), galvanostatic and potentiostatic charge/discharge measurements. The resulting rate capability, cycle life and the maximum amount of iodine that can be electrodeposited in the positive AC electrode (with self-discharge measurements) are shown in Fig. 4. We used an AC working electrode (diameter $=3 \mathrm{~mm}$ ) with a mass of $0.3 \mathrm{mg}$, an AC counter-electrode (diameter $=10 \mathrm{~mm}$ ) with a mass of $30 \mathrm{mg}$ and about $250 \mu \mathrm{L}$ electrolyte (three Whatman separators with $10 \mathrm{~mm}$ in diameter). This ensures that neither the total amount of $\mathrm{I}^{-}$in the cell nor the double-layer capacitance of the counter-electrode is limiting the $\mathrm{I}_{2}$ uptake in the AC WE pores.

\section{Data availability}

The data that support the findings of this study are available from the corresponding authors on request.

\section{Code availability}

The IgorPro (Wavemetrics) code used for in situ SAXS data analysis and stochastic modeling are available from the corresponding author C.P. on request.

Received: 3 April 2020; Accepted: 25 August 2020;

Published online: 24 September 2020

\section{References}

1. Larcher, D. \& Tarascon, J. M. Towards greener and more sustainable batteries for electrical energy storage. Nat. Chem. 7, 19 (2014).

2. Chun, S.-E. et al. Design of aqueous redox-enhanced electrochemical capacitors with high specific energies and slow self-discharge. Nat. Commun. 6, 7818 (2015).

3. Lee, J. et al. Redox-electrolytes for non-flow electrochemical energy storage: a critical review and best practice. Prog. Mater. Sci. 101, 46-89 (2019).

4. Frackowiak, E., Fic, K., Meller, M. \& Lota, G. Electrochemistry serving people and nature: high-energy ecocapacitors based on redox-active electrolytes. ChemSusChem 5, 1181-1185 (2012).

5. Evanko, B., Boettcher, S. W., Yoo, S. J. \& Stucky, G. D. Redox-enhanced electrochemical capacitors: status, opportunity, and best practices for performance evaluation. ACS Energy Lett. 2, 2581-2590 (2017).

6. Bai, C. et al. A sustainable aqueous Zn-I2 battery. Nano Res. 11, 3548-3554 (2018).

7. Li, Y., Liu, L., Li, H., Cheng, F. \& Chen, J. Rechargeable aqueous zinc-iodine batteries: pore confining mechanism and flexible device application. Chem. Commun. 54, 6792-6795 (2018).

8. Lee, J. et al. Nanoconfinement of redox reactions enables rapid zinc iodide energy storage with high efficiency. J. Mater. Chem. A 5, 12520-12527 (2017).

9. Xie, C., Zhang, H., Xu, W., Wang, W. \& Li, X. A long cycle life, self-healing zinc-iodine flow battery with high power density. Angew. Chem. Int. Ed. 57, 11171-11176 (2018).

10. Winsberg, J., Hagemann, T., Janoschka, T., Hager, M. D. \& Schubert, U. S. Redox-flow batteries: from metals to organic redox-active materials. Angew. Chem. Int. Ed. 56, 686-711 (2017).

11. Schranger, H., Barzegar, F. \& Abbas, Q. Hybrid electrochemical capacitors in aqueous electrolytes: challenges and prospects. Curr. Opin. Electrochem. 21, 167-174 (2020).

12. Zhang, S. et al. Naturally abundant high-performance rechargeable aluminum/iodine batteries based on conversion reaction chemistry. J. Mater. Chem. A 6, 9984-9996 (2018).
13. Tian, H. et al. High power rechargeable magnesium/iodine battery chemistry Nat. Commun. 8, 14083 (2017)

14. Huang, Q., Yang, J., Ng, C. B., Jia, C. \& Wang, Q. A redox flow lithium battery based on the redox targeting reactions between LiFePO4 and iodide. Energy Environ. Sci. 9, 917-921 (2016)

15. Wang, Y. L., Sun, Q. L., Zhao, Q. Q., Cao, J. S. \& Ye, S. H. Rechargeable lithium/iodine battery with superior high-rate capability by using iodine-carbon composite as cathode. Energy Environ. Sci. 4, 3947-3950 (2011).

16. Dané, L. M., Janssen, L. J. J. \& Hoogland, J. G. The iodine/iodide redox couple at a platinum electrode. Electrochim. Acta 13, 507-518 (1968).

17. Akkermans, R. P., Fulian, Q., Roberts, S. L., Suárez, M. F. \& Compton, R. G. Laser-activated voltammetry. Mechanism of aqueous iodide oxidation at platinum electrodes: theory and experiment. J. Phys. Chem. B 103, 8319-8327 (1999).

18. Abbas, Q., Fitzek, H., Schröttner, H., Dsoke, S. \& Gollas, B. Immobilization of polyiodide redox species in porous carbon for battery-like electrodes in eco-friendly hybrid electrochemical capacitors. Nanomaterials 9, 1413 (2019).

19. Przygocki, P., Abbas, Q., Babuchowska, P. \& Béguin, F. Confinement of iodides in carbon porosity to prevent from positive electrode oxidation in high voltage aqueous hybrid electrochemical capacitors. Carbon 125, 391-400 (2017).

20. Zhao, Y., Wang, L. \& Byon, H. R. High-performance rechargeable lithiumiodine batteries using triiodide/iodide redox couples in an aqueous cathode. Nat. Commun. 4, 1896 (2013).

21. Zhao, Q., Lu, Y., Zhu, Z., Tao, Z. \& Chen, J. Rechargeable lithium-iodine batteries with iodine/nanoporous carbon cathode. Nano Lett. 15, 5982-5987 (2015).

22. Bouchal, R., Boulaoued, A. \& Johansson, P. Monitoring polysulfide solubility and diffusion in fluorinated ether-based electrolytes by operando Raman spectroscopy. Batteries Supercaps 3, 397-401 (2020).

23. Stancovski, V. \& Badilescu, S. In situ Raman spectroscopic-electrochemical studies of lithium-ion battery materials: a historical overview. J. Appl. Electrochem. 44, 23-43 (2014)

24. Meng, Z. et al. Polyiodide-shuttle restricting polymer cathode for rechargeable lithium/iodine battery with ultralong cycle life. ACS Appl. Mater. Interfaces 10, 17933-17941 (2018).

25. Deplano, P., Ferraro, J. R., Mercuri, M. L. \& Trogu, E. F. Structural and Raman spectroscopic studies as complementary tools in elucidating the nature of the bonding in polyiodides and in donor-I2 adducts. Coord. Chem. Rev. 188, 71-95 (1999)

26. Svensson, P. H. \& Kloo, L. Synthesis, structure, and bonding in polyiodide and metal iodide-iodine systems. Chem. Rev. 103, 1649-1684 (2003).

27. Sharp, S. B. \& Gellene, G. I. Ab initio calculations of the ground electronic states of polyiodide anions. J. Phys. Chem. A 101, 2192-2197 (1997).

28. Milne, J. A Raman spectroscopic study of the effect of ion-pairing on the structure of the triiodide and tribromide ions. Spectrochim. Acta A 48 , 533-542 (1992).

29. Prehal, C. et al. Tracking the structural arrangement of ions in carbon supercapacitor nanopores using in situ small-angle X-ray scattering. Energy Environ. Sci. 8, 1725-1735 (2015).

30. Prehal, C. et al. Quantification of ion confinement and desolvation in nanoporous carbon supercapacitors with modelling and in situ X-ray scattering. Nat. Energy 2, 16215 (2017).

31. Prehal, C. et al. A carbon nanopore model to quantify structure and kinetics of ion electrosorption with in situ small angle X-ray scattering. Phys. Chem. Chem. Phys. 19, 15549 (2017).

32. Futamura, R. et al. Partial breaking of the Coulombic ordering of ionic liquids confined in carbon nanopores. Nat. Mater. 16, 1225 (2017).

33. Brousse, T., Bélanger, D. \& Long, J. W. To be or not to be pseudocapacitive? J. Electrochem. Soc. 162, A5185-A5189 (2015).

34. Ferrari, A. C. \& Robertson, J. Interpretation of Raman spectra of disordered and amorphous carbon. Phys. Rev. B 61, 14095-14107 (2000).

35. Das, A. et al. Monitoring dopants by Raman scattering in an electrochemically top-gated graphene transistor. Nat. Nanotechnol. 3, 210-215 (2008).

36. Przygocki, P., Abbas, Q. \& Béguin, F. Capacitance enhancement of hybrid electrochemical capacitor with asymmetric carbon electrodes configuration in neutral aqueous electrolyte. Electrochim. Acta 269, 640-648 (2018).

37. Barpanda, P., Fanchini, G. \& Amatucci, G. G. Structure, surface morphology and electrochemical properties of brominated activated carbons. Carbon 49, 2538-2548 (2011).

38. Abbas, Q., Fitzek, H., Pavlenko, V. \& Gollas, B. Towards an optimized hybrid electrochemical capacitor in iodide based aqueous redox-electrolyte: Shift of equilibrium potential by electrodes mass-balancing. Electrochim. Acta 337 135785 (2020)

39. Mulazzi, E., Pollini, I., Piseri, L. \& Tubino, R. Selective resonant Raman enhancement in polyiodide chains. Phys. Rev. B 24, 3555-3563 (1981). 
40. Naorem, H. \& Devi, S. D. Spectrophotometric determination of the formation constant of triiodide ions in aqueous-organic solvent or polymer mixed media both in absence and presence of a surfactant. Spectrochim. Acta A 101, 67-73 (2013).

41. Kleinberg, J. \& Davidson, A. W. The nature of iodine solutions. Chem. Rev. 42, 601-609 (1948).

42. Prehal, C., Koczwara, C., Amenitsch, H., Presser, V. \& Paris, O. Salt concentration and charging velocity determine ion charge storage mechanism in nanoporous supercapacitors. Nat. Commun. 9, 4145 (2018).

43. Prehal, C. et al. Comparing pore structure models of nanoporous carbons obtained from small angle X-ray scattering and gas adsorption. Carbon 152, 416-423 (2019).

44. Saurel, D. et al. A SAXS outlook on disordered carbonaceous materials for electrochemical energy storage. Energy Storage Mater. 21, 162-173 (2019).

45. Gommes, C. Three-dimensional reconstruction of liquid phases in disordered mesopores using in situ small-angle scattering. J. Appl. Crystallogr. 46, 493-504 (2013).

46. Kwak, W.-J. et al. Lithium-oxygen batteries and related systems: potential, status, and future. Chem. Rev. 120, 6626-6683 (2020).

47. Pang, Q., Liang, X., Kwok, C. Y. \& Nazar, L. F. Advances in lithium-sulfur batteries based on multifunctional cathodes and electrolytes. Nat. Energy 1, 16132 (2016).

48. Katumitu, H. \& Sumio, N. The detemination of the formation constants of the triiodide ion in water-alcohol mixed solvents at various temperatures. Bull. Chem. Soc. Jpn 50, 566-569 (1977).

49. Pauw, B. R. Everything SAXS: small-angle scattering pattern collection and correction. J. Phys. Condens. Matter 25, 383201 (2013).

50. Gommes, C. J. Stochastic models of disordered mesoporous materials for small-angle scattering analysis and more. Microporous Mesoporous Mater. 257, 62-78 (2018).

51. Ruland, W. Small-angle scattering of two-phase systems: determination and significance of systematic deviations from Porod's law. J. Appl. Crystallogr. 4, 70-73 (1971)

52. Gommes, C. J. \& Roberts, A. P. Structure development of resorcinolformaldehyde gels: microphase separation or colloid aggregation. Phys. Rev. E 77, 041409 (2008).

53. Roberts, A. P. Statistical reconstruction of three-dimensional porous media from two-dimensional images. Phys. Rev. E 56, 3203-3212 (1997).

54. Berk, N. Scattering properties of the leveled-wave model of random morphologies. Phys. Rev. A 44, 5069 (1991).

\section{Acknowledgements}

Q.A. acknowledges funding by the Austrian Science Fund (FWF) under the Lise Meitner project M 2576-N37 and thanks Martin Wilkening (ICTM) for the advisory support. S.A.F. and C.P. are indebted to the European Research Council (ERC) under the European Union's Horizon 2020 research and innovation program (grant agreement no. 636069) and the Austrian Federal Ministry of Science, Research and Economy and the Austrian Research Promotion Agency (grant No. 845364). H.F. acknowledges the Graz Centre for Electron Microscopy (ZFE) and Hartmuth Schröttner for financial support through the HRSM-Project ELMINet Graz (Austrian Ministry of Education). V.P. thanks
Eduard Arzt (INM) for his continuing support. The authors acknowledge TU Graz for support through the Lead Project LP-03. Likewise, the use of SOMAPP Lab, a core facility supported by the Austrian Federal Ministry of Education, Science and Research, the Graz University of Technology, the University of Graz, and Anton Paar GmbH is acknowledged. We thank EL-Cell GmbH (Hamburg, Germany) for providing the ECC Opto-Gas cell for in situ Raman and Anton Paar GmbH (Graz, Austria) for the joint in situ SAXS cell development

\section{Author contributions}

C.P. carried out in situ scattering experiments, the corresponding data analysis and the stochastic modeling. H.F. conducted in situ Raman experiments and data analysis (supported by Q.A. and C.P.). C.P. and Q.A. carried out electrochemical measurements. C.P., Q.A., H.F. and S.A.F. conceptualized the work. C.P wrote the initial version of the manuscript. C.P., H.F., G.K., V.P., B.G., S.A.F. and Q.A. contributed to results interpretation and revising the manuscript.

\section{Competing interests}

The authors declare no competing interests.

\section{Additional information}

Supplementary information is available for this paper at https://doi.org/10.1038/s41467 020-18610-6.

Correspondence and requests for materials should be addressed to C.P., S.A.F. or Q.A.

Peer review information Nature Communications thanks Xuehang Wang, and the other, anonymous, reviewer(s) for their contribution to the peer review of this work. Peer reviewer reports are available.

Reprints and permission information is available at http://www.nature.com/reprints

Publisher's note Springer Nature remains neutral with regard to jurisdictional claims in published maps and institutional affiliations.

(c) (i) Open Access This article is licensed under a Creative Commons By Attribution 4.0 International License, which permits use, sharing, adaptation, distribution and reproduction in any medium or format, as long as you give appropriate credit to the original author(s) and the source, provide a link to the Creative Commons license, and indicate if changes were made. The images or other third party material in this article are included in the article's Creative Commons license, unless indicated otherwise in a credit line to the material. If material is not included in the article's Creative Commons license and your intended use is not permitted by statutory regulation or exceeds the permitted use, you will need to obtain permission directly from the copyright holder. To view a copy of this license, visit http://creativecommons.org/ licenses/by/4.0/.

(C) The Author(s) 2020, corrected publication 2020 